Available online at www.sciencedirect.com

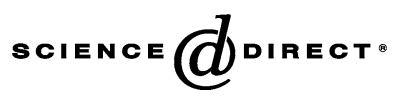

EPSL

Earth and Planetary Science Letters xx (2004) xxx- xxx

www.elsevier.com/locate/epsl

\title{
Error bars for the global seismic $Q$ profile
}

\author{
Joseph Resovsky*, Jeannot Trampert, R.D. Van der Hilst ${ }^{1}$ \\ Department of Earth Sciences, Utrecht University, PO Box 80021, 3508 TA Utrecht, the Netherlands
}

Received 30 July 2004; received in revised form 1 December 2004; accepted 8 December 2004

Editor: S. King

\begin{abstract}
The radial attenuation profile of the Earth is needed to account for dispersion effects when interpreting seismic velocities and can provide important constraints on composition. To date, most radial $Q$ models have been produced using traditional damped inversions of free oscillation and surface wave data. Because such inversions can severely underestimate the model uncertainties that are needed to guide mineralogical and dynamic interpretation, and because the quality of data has continued to improve, we revisit this seismic inverse problem using a model space search approach already proven effective with similar data. We do, indeed, observe model uncertainties at least an order of magnitude greater than earlier estimates. At the same time, we find that $Q$ is determined well enough to confirm that the data favor several important features previously disputed because of questions of consistency. These include shear attenuation that drops significantly in the lower third of the lower mantle and bulk attenuation that is negligible in the inner core but stronger in the outer core and lower mantle than suggested by most models. (C) 2004 Elsevier B.V. All rights reserved.
\end{abstract}

Keywords: seismic attenuation; neighbourhood algorithm

\section{Introduction}

Of the many forms of seismic constraints on deep Earth composition and dynamics, the radial attenuation profile (attenuation as a function only of depth) is perhaps the simplest that is of significant interest

\footnotetext{
* Corresponding author. Tel.: +3130253 5138; fax: +31 30 2533486 .

E-mail addresses: resovsky@geo.uu.nl (J. Resovsky), jeannot@geo.uu.nl (J. Trampert).

${ }^{1}$ Earth Atmospheric and Planetary Sciences, Massachusetts Institute of Technology, Cambridge, MA, USA.
}

while remaining poorly constrained. The inverse problem for attenuation (or its multiplicative inverse, $Q$ ) is simple, in principle, because the primary data are a few hundred measurements of surface wave (SW) and free oscillation (FO) attenuation that are linear functionals of the $Q$ profile of the Earth. The significance of the problem is that the attenuation profile informs models of composition and dynamics by providing a direct constraint upon which dissipative processes are dominant at a given depth. These include constraints on the presence of partial melt in the upper mantle transition zone and on phase changes near the inner core boundary. The $Q$ profile,

0012-821X/\$ - see front matter (C) 2004 Elsevier B.V. All rights reserved. doi:10.1016/j.eps1.2004.12.008 
particularly in the upper mantle, is also needed to make dispersion corrections of seismic velocities before they are interpreted in terms of composition [1], or dynamics [2].

This kind of interpretation relies upon knowledge not only of an attenuation model that best fits the seismic data, but also of the range of models that are reasonably consistent with the data, or the model uncertainty. It is here that the problem of constraining attenuation manifests itself. Commonly used radial $Q$ models PREM [3], QM1 [4] and QL6 [5] have stated uncertainty in shear attenuation of only a couple of percent in most of the mantle but differ from one another by $10-50 \%$, so that they are formally inconsistent. This situation is created both by the poor signal-to-noise characteristics quantified by the rather large data errors, and by the use of inconsistent data sets contaminated by inadequately described bias.

Firstly, the seismic amplitude signal that yields attenuation measurements is strongly affected by elastic focusing, seismic noise and signal processing errors. This makes the measurements very noisy and weakens the resolving power of the data enough that even simple model parameterizations, such as that used for PREM, produce effectively underdetermined inverse problems. Straightforward inversion of the data must, therefore, be performed with strong damping, which has been shown to produce badly underestimated model uncertainty [6]. It is important to improve the model error estimates because features that appear robust in some models, like the decreasing $Q$ with depth in the lower mantle of QM1, may not be resolvable if model uncertainties approach the $50 \%$ level of the differences among existing models. This is why we adopt a model space search approach, in which neither the best fit solutions nor model uncertainties are biased by a priori choices of damping.

Secondly, it has been shown that SW $Q$ measurements can be biased low at long periods by difficulties in defining windows that isolate modes [7], while FO $Q$ measurements can be biased high by the relatively high noise levels in the latter part of the long time series used for this kind of analysis [8,9]. These biases at least partly explain the large and well documented [7-10] discrepancy between SW and FO measurements of fundamental modes, and between the radial $Q$ models that result from inverting these data. It remains unclear, however, just how much model uncertainty may be introduced by these biases, because observed differences in best fit models may actually represent variation within a range of models providing acceptable fits to both data sets. By mapping out these model ranges, this study provides a more complete assessment of possible bias in the models.

A final reason to employ a model space search is to map out tradeoffs among different model parameters. This is particularly important in trying to extract information about bulk attenuation. Resolution of $Q_{\kappa}$ is so poor that several authors $[5,11]$ have resorted to crude model space searches in the form of inversions with different single-layer $Q_{\kappa}$ parameterizations. This has led to a wide variety of locations for the bulk attenuation required to fit measurements of radial modes, including the upper mantle (QL6), the inner core (PREM), and combinations of these and other layers $[10,12]$. By mapping tradeoffs, we can discover which features are favored by the data without needing to resolve each parameter.

\section{Method}

Our primary enhancement of attenuation modeling comes through the method that we apply to the inverse problem, although we also use somewhat improved versions of the SW and FO data sets. For a given model of the radially symmetric distribution of density and elastic moduli within the earth, the linear inverse problem for attenuation has the form

$q_{i}=\int_{0}^{r_{\mathrm{E}}}\left[K_{i}(r) \kappa(r) q_{\kappa}(r)+M_{i}(r) \mu(r) q_{\mu}(r)\right] r^{2} \mathrm{~d} r$,

where $K_{i}$ and $M_{i}$ are analytically determined [13] data kernels that describe the physical theory of how radial variation in bulk $(\kappa)$ and shear $(\mu)$ attenuation is expressed in the seismic attenuation data $q_{i}$. The usual approach is create a matrix from equations of this form for all the data to be used and to invert that matrix to find a model that "best" fits the data. Though this approach is computationally efficient, it has the disadvantage of requiring a priori information in the form of strong damping to compensate for the relatively poor resolution of the existing attenuation data set. If a Backus-Gilbert regularization technique 
$[14,15]$ is applied, optimal use can be made of the data resolution, but model uncertainty still tends to be underestimated, and model features that come from the data rather than the regularization are best determined by performing many inversions with different regularization schemes $[6,11,16]$.

Instead, we choose the forward modeling approach, in which the kernels are used to create synthetic data from many different possible models. For each model $\mathbf{m}$, we record the mean misfit of the synthetic data set, defined for $N$ attenuation data $q_{i}=1 / Q_{i}$ with errors $\sigma_{i}$ as

$\chi_{\text {mean }}^{\mathbf{m}}=\left[\sum_{i=1}^{N} \frac{\left(q_{i}^{\mathbf{m}}-q_{i}^{\mathrm{data}}\right)^{2}}{N \sigma_{i}^{2}}\right]^{1 / 2}$.

This misfit is used to assign a multidimensional posterior probability density to the corresponding
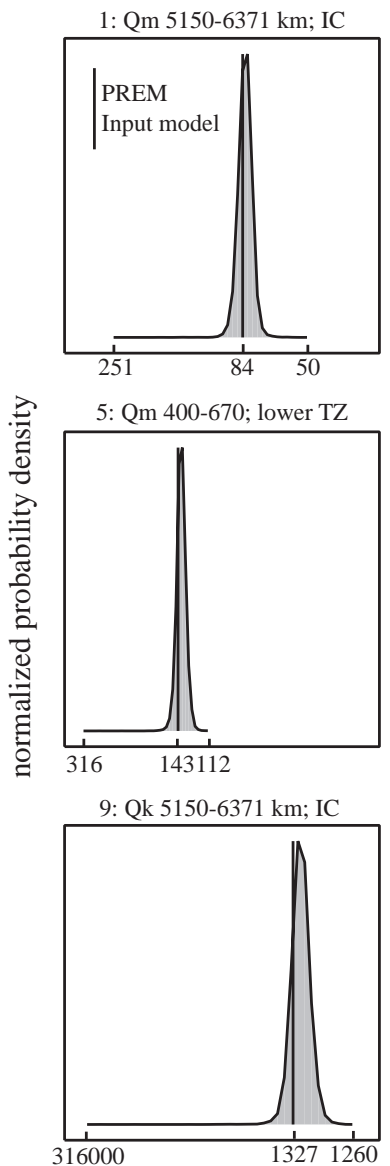

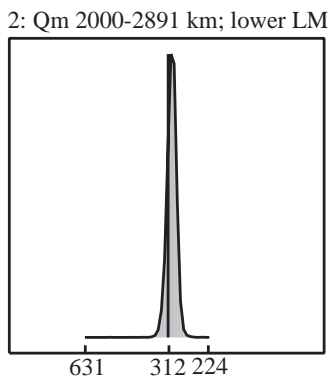

6: Qm 220-400 km; upper TZ

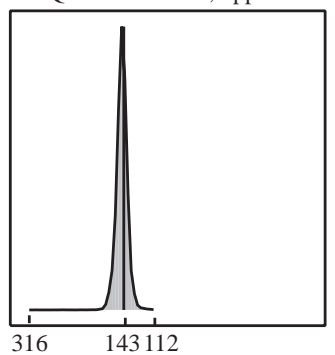

10: Qk 2891-5150 km; OC

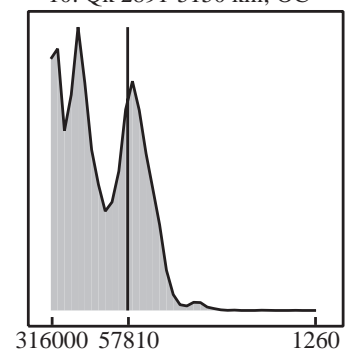

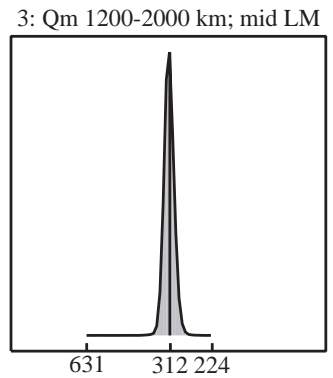

7: Qm 80-220 km; LVZ

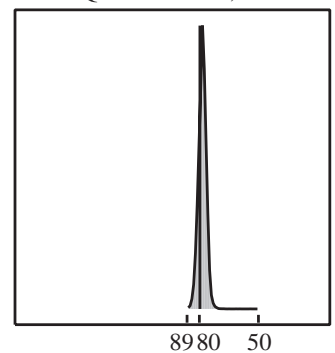

11: Qk 670-3480 km; LM

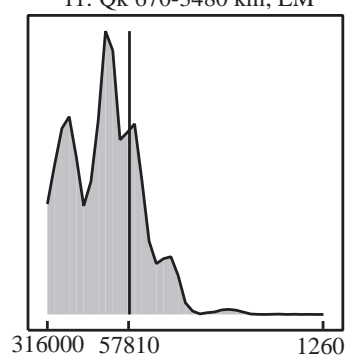

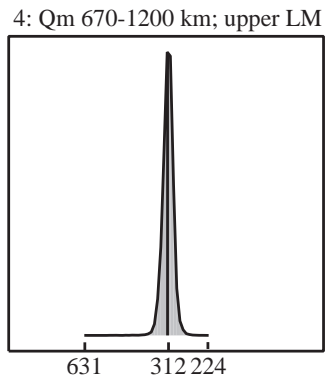

8: Qm 3-80 km ; LID

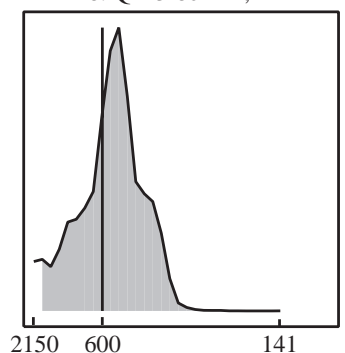

12: Qk 3-670 km; UM

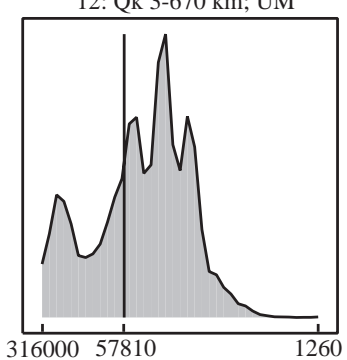

Fig. 1. 1D PDFs for a synthetic experiment matching the data, model parameterization and NA search parameters of our real model space search. The input model is PREM. To the synthetic data, we add synthetic noise using random deviates drawn from Gaussian distributions that match the (adjusted) error in our real data set. The probability densities have been normalized using the overall maximum and the $Q$ axes are logarithmic. The $Q$ axis limits shown are those used in the first (wide) stage of our model space search, while the PDF endpoints (indicated in the figure) show the range of the second (narrower) search. The $Q$ ranges for the initially wider search are: panel 1 [5012-32]; panels 2, 3 and 4 [1000-100]; panels 5, 6 and 7 [465-21]; panel 8 [2150-215]; panels 9, 10, 11 and 12 [316,000-316]. The input model is everywhere within the high-likelihood region. Due to added data errors, the input model does not coincide with the most likely model, the difference being what is expect from the magnitude of the data error. The widths of the peaks illustrate the resolving power of the data explaining the known scatter of existing models. These results confirm that the NA search is correctly tuned and the data provide adequate resolution for the chosen parameterization. 
point in model space, defined by $\mathbf{P}(\mathbf{m})=\exp \left(-\chi^{2} / 2\right)$ for presumed Gaussian error statistics in the data [17]. From this multidimensional probability density function (PDF), numerical integration can be used to project the 1D PDFs that provide more complete descriptions of uncertainty in individual model parameters than do model error bars, as well as 2D PDFs that provide the complete description of covariances among parameters. Even with Gaussian data errors, the existence of both data and model null spaces implies that the PDFs themselves are not necessarily either Gaussian or single peaked [18].

The challenge of this approach is to find a tool for sampling model space in such a way that all significant regions of good and poor fit are reliably mapped out with as few models as possible, while allowing for rapidly converging numerical integration of PDFs. One such tool is provided by the neighbourhood algorithm (NA), a model space search and Bayesian assessment procedure [17,19]. In NA, the mean misfit of an existing model is used to assign a constant PDF to the model space subset (neighbourhood) of points closer to that model than to any other existing model. At each iteration of the search phase of NA, a specific number $n_{\mathrm{r}}$ of new models are randomly selected from the neighbourhoods of the best $n_{\mathrm{b}}$ existing models. To complete the iteration, the mean misfits of the new models are computed. Once the search is completed, random walks guided by the neighbourhood PDFs perform the Bayesian numerical integrations that produce the global PDFs. NA has already been successfully applied to an inverse problem very similar to the present one $[6,20]$. Synthetic experiments, such as that shown in Fig. 1, confirm that NA can also be applied with confidence to attenuation data. The resulting PDFs correctly place the input model in the region of greatest probability density. The difference between the distribution peaks and the input model is no more than what should result from the synthetic data error. At the same time, the PDFs identify a pattern of broad and narrow likelihood peaks that agree with the "true" model uncertainty suggested by the ranges of published models [10]: a much wider range for $Q_{\kappa}$ than $Q_{\mu}$, the widest range of $Q_{\mu}$ values occurring in the uppermost mantle and the narrowest range of $Q_{\kappa}$ values occurring in the inner core. The NA forward modeling approach thus appears to be completely appropriate for describing the Earth's attenuation profile.

\section{Data}

We employ a data set of $570 \mathrm{SW}$ and FO attenuation measurements recorded at the Reference Earth Model web page [21] as of 1 January 2004. These include SW fundamental mode measurements [7,22-24], FO fundamental data [25], FO overtone and longer period fundamental measurements [26-29] and some unpublished updates of these data. The unpublished data attributed to "Um" on the REM pages have anomalously low uncertainties, which, given that there is no documentation to suggest that a superior technique was used, have been multiplied by a factor of 5.0 to provide consistency with the other data. Similarly, uncertainties for the $\mathrm{He}$ and Tromp[27] measurements were multiplied by 2.0 (because of errors known to be caused by the use of too few events [28]), those for Resovsky and Pestana (2002) by 3.0 (based on our familiarity with those data), and those for the inconsistent 150-300 s SW and FO measurements of spheroidal fundamentals by 3.0. Except for the latter adjustment, which is discussed below, these changes improve model misfits without altering the other characteristics of the model space.

We exclude only the data for multiplet ${ }_{10} S_{2}$, which is extremely sensitive to the location of the inner core boundary in the reference model used to describe the eigenfunctions. Although body wave data can be important in constraining seismic attenuation, even in the frequency band below $100 \mathrm{mHz}$ dominated by surface waves and normal modes, the latter types of data have by far the best resolution, and have been relied upon exclusively by most inversions to date. Incorporation of body wave data in our model space searches is the subject of future research and may well require the exploration of frequency-dependent $Q$ models.

As mentioned previously, SW and FO measurements of spheroidal (Rayleigh wave) fundamentals in the 150-300 s period range are inconsistent, forming two self-consistent trends separated by a gap of width 3-5 times the stated measurement uncertainties. The modeling tradeoffs that result have already been investigated [5] and we choose not to map them out 
again. Instead, we observe that several studies [7-9] have argued for the correction of both data sets in the direction of their mean, with larger uncertainties. This can be accomplished in the inverse problem by simply multiplying the data error for these measurements by a large enough factor to bring them into formal agreement with one another. In this way, we make the 150 $300 \mathrm{~s}$ spheroidal fundamental modes less important relative to data from overtones and from the high $Q$ fundamentals, with periods longer than $300 \mathrm{~s}$ that are less subject to noise biasing. The latter kind of data has been greatly improved with generalized spectral fitting analysis of the Coriolis-coupled fundamentals in the 300-600 s band [28]. We also test our results for sensitivity to this approximation by performing "reduced" data searches in which only one of the two controversial data sets is included, with its originally stated error.

\section{Parameterization}

Because radial variations in attenuation can extend over several orders of magnitude, we have found it most convenient to parameterize our models in terms of $\log (q)$. This is further justified by our observation that model likelihood distributions are more nearly log-normal than normal. For ease in examining tradeoffs in sensitivity to $Q$ at different depths and because we are doubtful of our ability to resolve steep attenuation gradients within the established mantle layers, we choose to work with layered models. These are parameterized as the average of $Q_{\mu}$ or $Q_{\kappa}$ over a given layer. Despite appearances, layered models neither enforce nor imply discontinuities between layers. Layer averages even for smooth models will appear discontinuous. A more legitimate concern with layered models is that the bounds for the average of $Q$ within a layer can underestimate the bounds of $Q$ itself when strong gradients exist within the layer. This could be the case if there is a very sharp drop in $Q$ in the lowermost $500 \mathrm{~km}$ of the mantle [12]. However, recent smooth models (e.g. QM1 [4] and Q7U15L [5]) achieve good data fit with only modest gradients, so we can be reasonably confident that strong gradients will not be a problem.

Since our data set is not unlike that which informed the PREM parameterization, we employ the five $Q_{\mu}$ layers of that model as a template, and then add an upper transition zone layer (matching that layer in the PREM velocity model), and break the lower mantle into three roughly equal layers. The former addition allows us to look for transition zone $Q$ gradients such as those of QM1, while the latter three layers are sufficient to examine the question of whether lower mantle $Q$ mostly declines with depth (QM1), peaks in the mid-mantle (Q7U15L [5] and PAR3P [11]), mostly increases with depth [12], or is flat (PREM and QL6) within modeling uncertainty. We also include four $Q_{\kappa}$ layers so that we can provide better answers to the question of where seismic data favor the placement of bulk attenuation, though we do not expect to resolve these layers completely independently.

We have, then, 12 model parameters, which are identified in Fig. 1. The endpoints of the PDFs shown in Fig. 1 represent the a priori bounds of the final model space search that produced the results in the subsequent figures. Our initial search was conducted, with a less dense sampling, in a larger range, indicated by the $Q$ axis limits of that figure, to include all previous published models. Parts of the model space

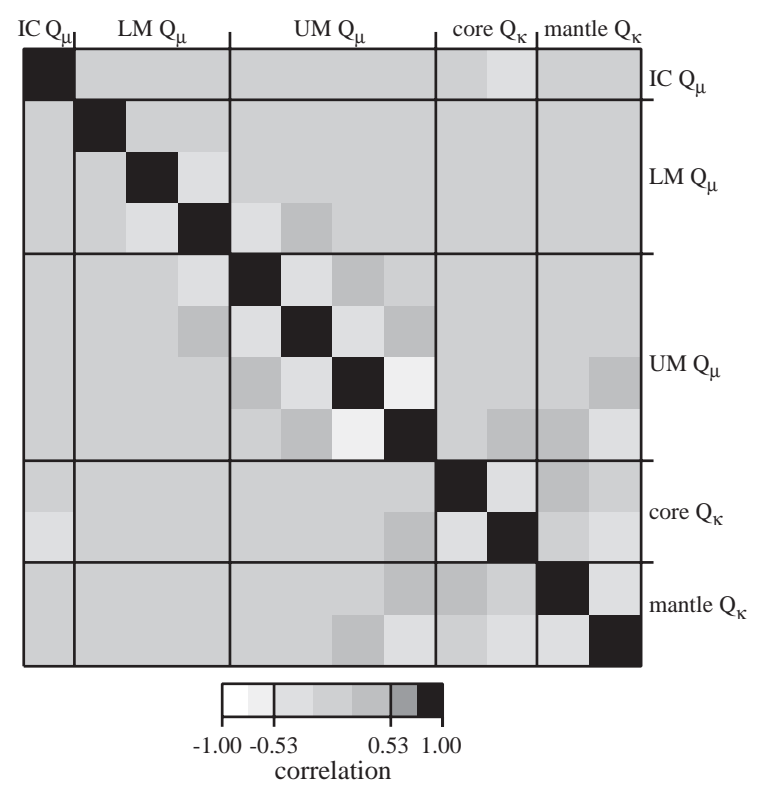

Fig. 2. Correlation matrix displaying observed tradeoffs in fit to the data for our model parameters. The correlation $c_{i j}$ between parameters $i$ and $j$ is a normalization of the covariance $v_{i j}$ defined by $c_{i j}=v_{i j} / \sqrt{v_{i i} v_{j j}}$. The largest off-diagonal element is the $-61 \%$ correlation for $Q_{\mu}$ in the top two layers. 
that produced negligible PDFs were excluded from the final search to improve computational efficiency. The final model search phase of the NA mapping is performed by choosing 1000 models from the 1000 best neighbourhoods for 300 iterations, while the Bayesian phase uses the search phase results to guide 5 random walks of 5000 steps.

As described in Section 2, the results in Fig. 1 demonstrate that the data are adequate to resolve models with the selected layers, using the above search parameters. The model correlation matrix that results from model space mapping using the real data, shown in Fig. 2, confirms that tradeoffs between model parameters are modest for our chosen parameterization. Even strong tradeoffs would not render models uninterpretable, given our ability to investigate the full 2D marginals that produce the tradeoffs (Fig. 3). However, that tradeoffs are relatively small makes the interpretation of attenuation profiles more straightforward than this otherwise would be.

\section{Results}

With our model space search, we were able to find a most likely family of models that provide
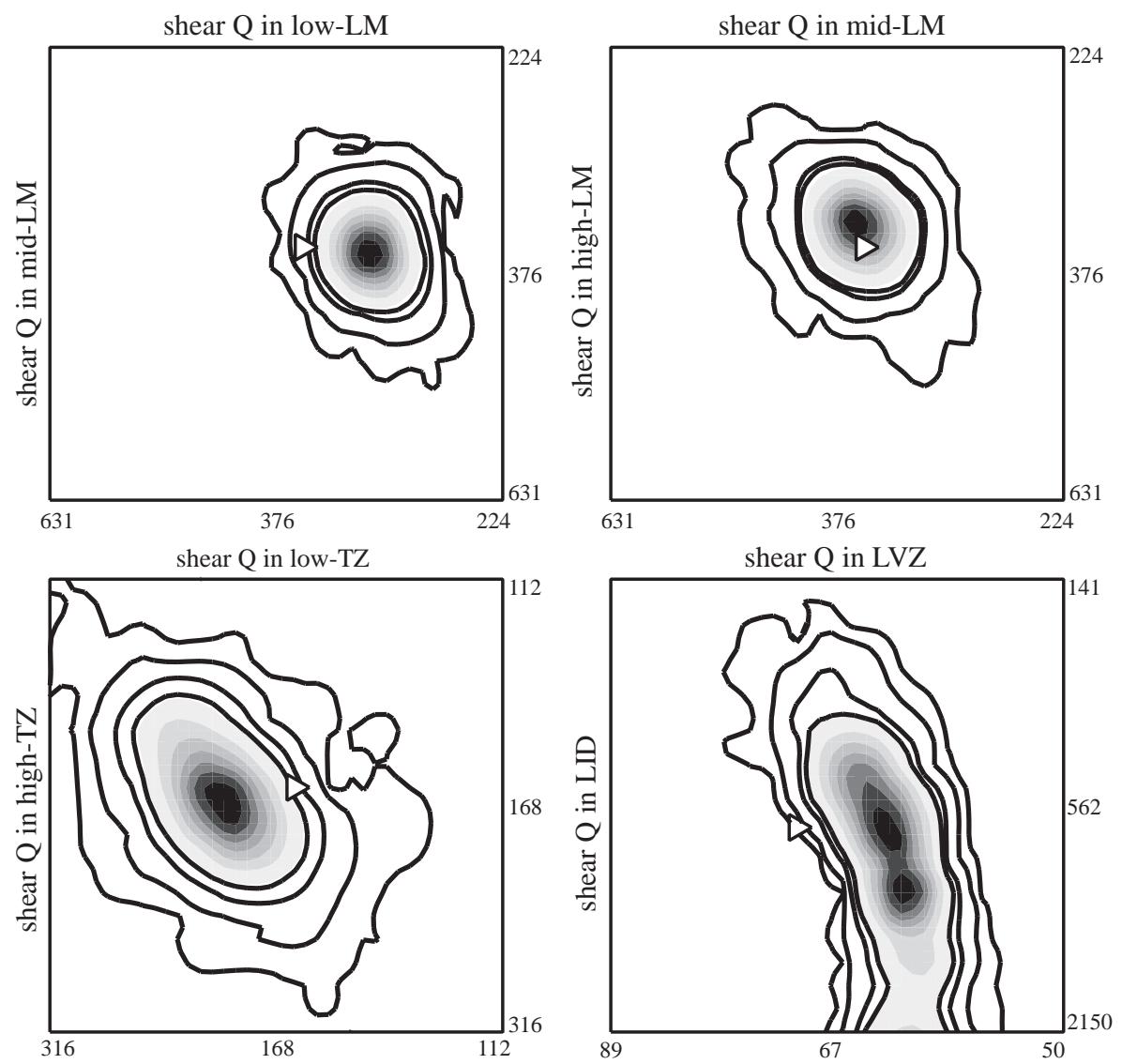

Fig. 3. 2D PDFs corresponding to four of the elements of Fig. 2, showing details of some tradeoffs. Contour lines indicate outward increasing confidence levels with the total enclosed likelihood; they correspond to $80 \%, 90 \%, 95 \%$ and $99 \%$. Increasing likelihood, on the other hand, is shown with darker shading. The white triangle corresponds to PREM. Random deviates drawn from these marginals can be used to find the likelihood that two layers have significantly different mean attenuation. The strong negative correlation for $Q_{\mu}$ in the LVZ and LID visible in Fig. 2 is as attributable to the relatively small variance for the former layer as to the overall slope of the corresponding PDF. The tradeoff between the two TZ layers is much more pronounced, so that any gradient between them is difficult to resolve. 
data fits comparable the results of the Durek and Ekström [5] modeling efforts. After removing three apparently anomalous records from an original set of 239 measurements, they achieved a $\chi$-misfits (Eq. (2)) between 1.10 and 1.20 for various $Q_{\kappa}$ parameterizations. When we remove from our set of 570 data a subset of 9 anomalously poorly fit measurements (for which other data for the same modes is well fit), our best $\chi$-misfit is 1.16. In Fig. 4, we show a subset of the data used, together with corresponding predictions of QL6, of our best fitting model, and of 1000 models representing the model PDFs of Figs. 5 and 6. Just as in the QL6 inversions, we obtain good fits to most data, including funda- mentals and overtones, spheroidal and toroidal modes, and radial modes. Additional figures showing fits to the data may be obtained upon request from the authors.

The data subset of Fig. 4 is chosen because it displays the two primary differences in our fits to the data and those provided by QL6: that we obtain excellent fits to the spheroidal fundamentals in the 300-600 s Coriolis coupling band, and that our models tend to "split the difference" between the SW and FO trends below 300 s period. It appears that the latter result is a consequence of the former, rather than a consequence our use of both the SW and FO data below $300 \mathrm{~s}$. Models that fit the 300-600 s band

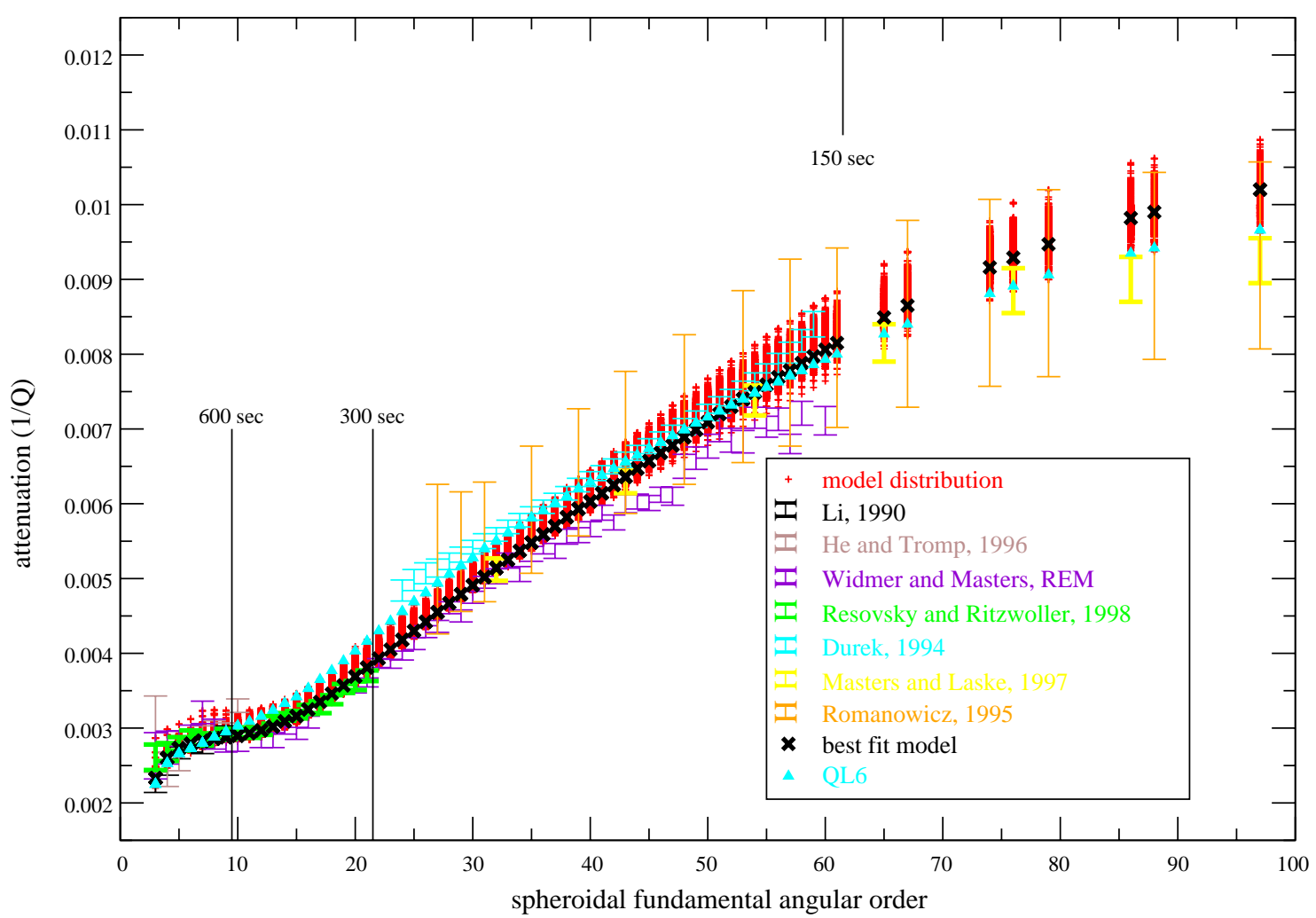

Fig. 4. Predictions for a representative sampling of models (small red crosses) compared to the data (error bars) for Rayleigh fundamentals. The data sources are color coded by source, including some from the Reference Earth Model (REM) web page that are either unpublished or refinements of published data. Also for comparison, the predictions of QL6 are shown with solid triangle and predictions of our best fitting are shown as black crosses. The sampling is a set of 1000 random deviates draw from the model PDFs projected in Figs. 3, 5 and 6 . We note that asymmetry about the best fit results from the distributions being approximately log-normal in attenuation. In the case of inconsistent data, our models usually "split the difference". The 150-300 s spheriodal fundamentals are an example of this. The 300-600 s fundamentals are better fit by our models than by QL6. For other mode subsets, our best fit predictions and those of QL6 are still more similar, our predictions show the same level of agreement with the data and the model sampling produces a similar range of predictions. 


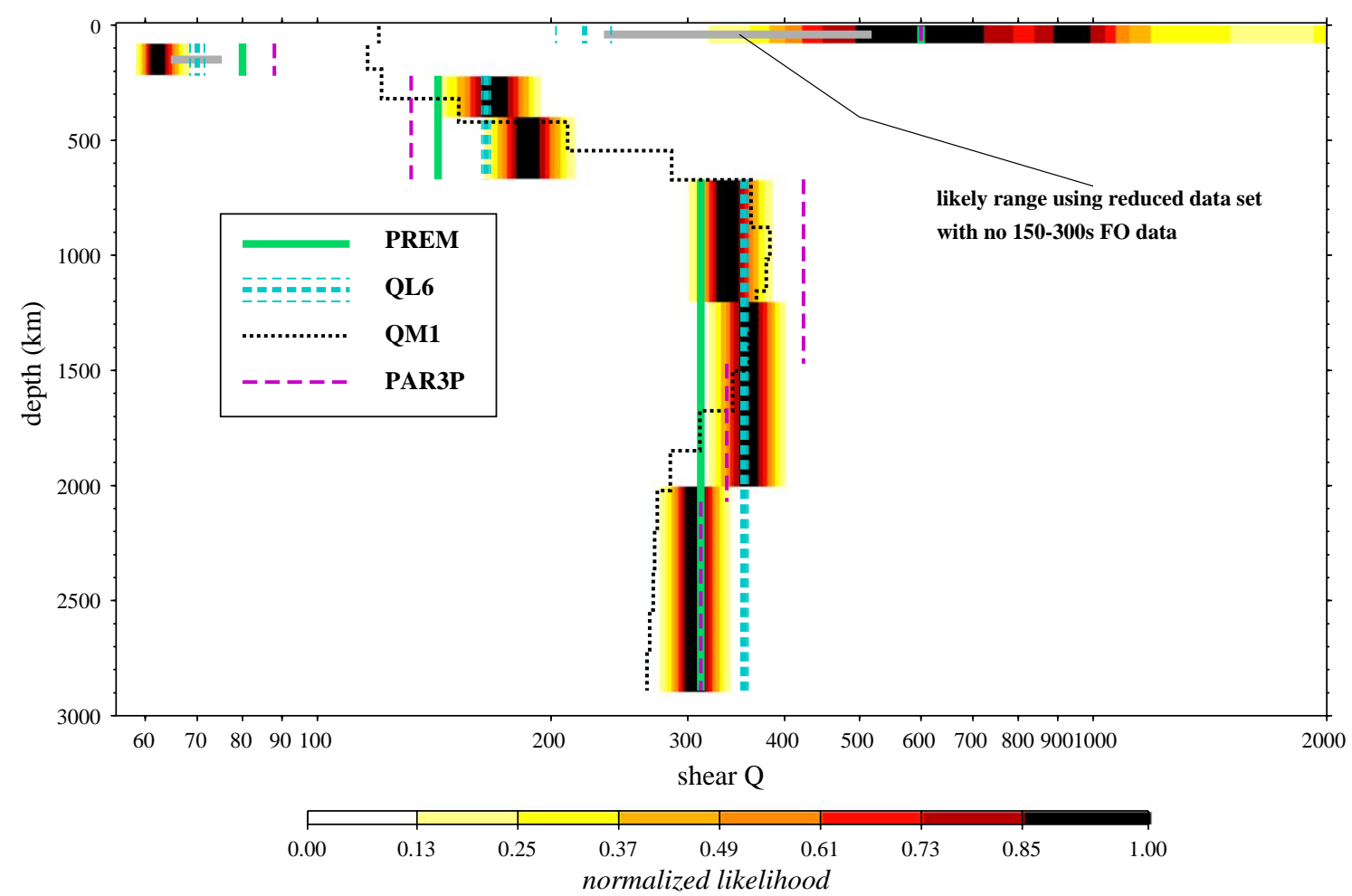

Fig. 5. Output 1D PDFs for $Q_{\mu}$ displayed as a radial profile together with some earlier models. Each individual marginal is unit normalized, so that the 1/e (orange) and 1/ $e^{2}$ (yellow) contours correspond to 1- $\sigma$ and 2- $\sigma$ model error bars. Note that the given error bars for model QL6, typical of error estimates for inversions, are much narrower. For the top two layers only, the results of a model space search with a different data set are shown as grey bars spanning the $2-\sigma$ range. The inner core layer results, not shown, may be found in the text.

and "split the difference" are favored even we use the "reduced" data set which does not include the 150 $300 \mathrm{~s}$ FO data. It is again important to note that the Resovsky and Ritzwoller [28] data that dominate the $300-600 \mathrm{~s}$ band were not available at the time of the QL6 inversions.

As illustrated by Figs. 5 and 6, the most likely mantle attenuation models (black regions in the model distributions) from our model space search have more in common with QL6 and its related models [5] than with other models. The inconsistencies with QL6 in the uppermost mantle are caused by our fitting of both the surface wave and free oscillation data below $300 \mathrm{~s}$, while QL6 fits the surface wave data only. When we repeat the search using our "reduced" data set with no 150-300 s FO data, the 2- $\sigma$ output ranges move into agreement with QL6, despite the continued influence of the Coriolis-coupled modes not included in the QL6 data set.
The increasing $Q_{\mu}$ through the $\mathrm{TZ}$ and top half of the LM, and the drop in the bottom half, are better matched by the more finely parameterized PAR3P and QM1 than by PREM and QL6, but most closely matched by the radially smooth model Q7U15L that accompanied QL6. This model was rejected by its authors because the extra gradients in $Q$ did not improve upon the data fit of QL6 [5]. But even if this is true, gradients can prove to be likely if there are more models with gradients than without gradients that provide similarly good fits. Our 2D PDFs (Fig. 3) allow us to show that this is the case. Drawing random deviates from the LLM-MLM PDF reveals $Q_{\mu}$ decreasing with depth in $95 \%$ of the models and drops of more than $15 \%$ in magnitude in more the $60 \%$ of models. The increase in the TZ is less strongly favoured, occurring in $75 \%$ of models, with increases of $5 \%$ or more only $59 \%$ of the time. The increase through the top half of the lower mantle was still less 


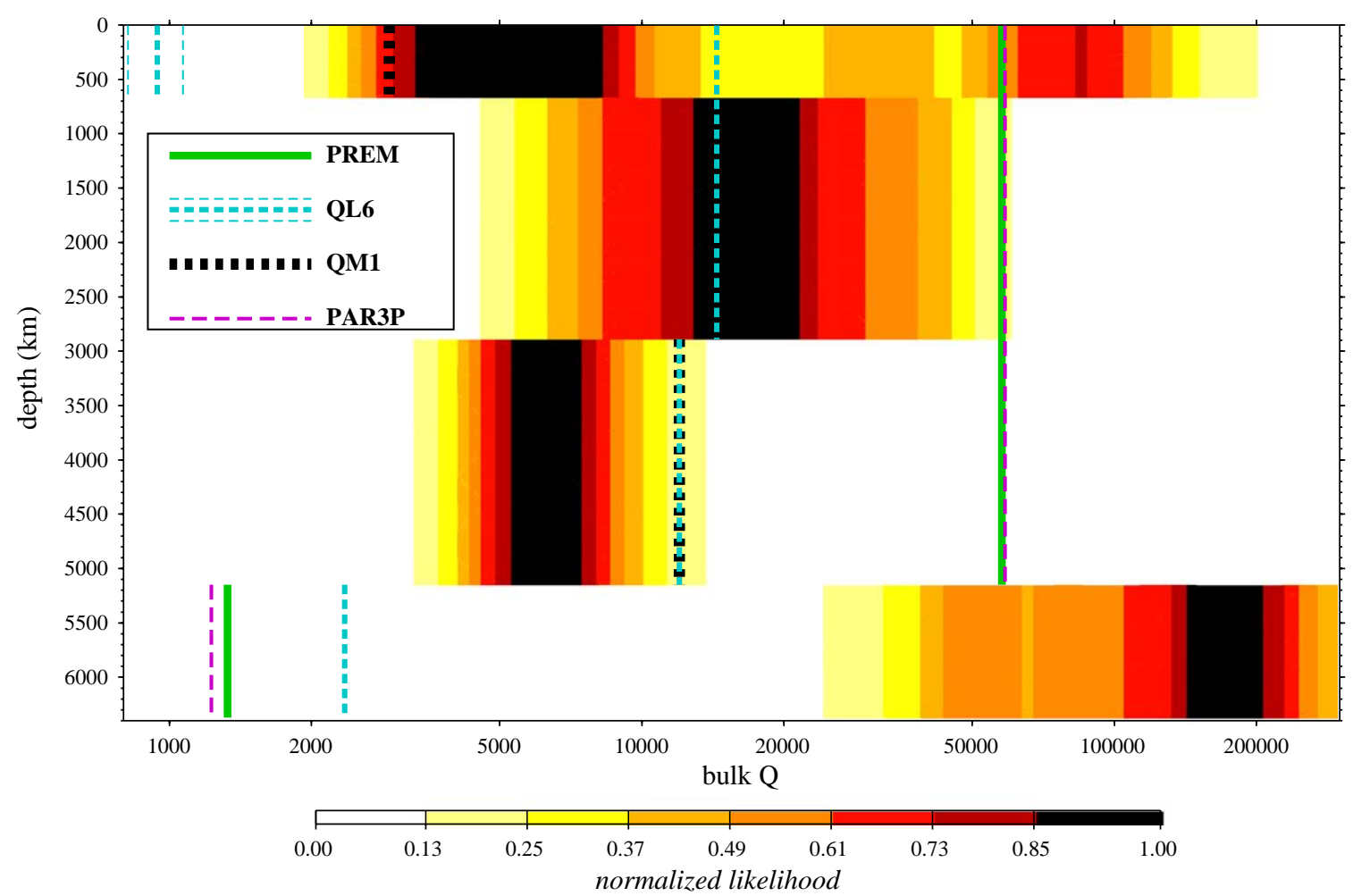

Fig. 6. Output 1D PDFs for $Q_{\kappa}$, displayed as in Fig. 5. Here, model QL6 is represented by four models: the preferred $Q_{\kappa}$ model, which is confined to the upper mantle, and three alternative models from the same study with $Q_{\kappa}$ distributed through the whole mantle or confined to the inner or outer core.

robust, occurring in only $63 \%$ of models, with a slight decrease becoming favored when we use the "reduced" data set. The strong decrease with depth in the top half of the lower mantle found by some inversions $[11,30]$ appears unlikely. When we perform searches in model spaces with more lower mantle layers, tradeoffs become severe enough that the gradients cannot be located more precisely than implied by the changing layer averages in Fig. 5 . For shear attenuation in the inner core, our normalized PDF values are above $85 \%$ for $90 \leq Q_{\mu} \leq 100$, above $37 \%$ (corresponding to the $1-\sigma$ range in a normal distribution) between 83 and 103, and above 13\% (2$\sigma)$ between 78 and 110 . This implies consistency with PREM (84.6), QL6 (104 \pm 8\%) and QM1 (109-112) albeit with error bars twice as wide as in QL6.

The distributions of likely $Q_{\kappa}$ values in the mantle are very wide, but clearly favor more bulk attenuation than included in PREM. This is not concentrated in the upper mantle as in the favored parameterization of
QL6 (shown with error bars), but distributed through the mantle as in one of the slightly less successful $Q_{\kappa}$ parameterizations of that study. Also in that study, the outer core was the second most successful location of bulk attenuation, and we find it to be the layer with the narrowest distribution and smallest mean $Q_{\kappa}$.

We confirm the Durek and Ekstrom [5] finding, contrary to PREM, that the inner core is the least likely location for bulk attenuation. The QL6 variant with $Q_{\kappa}$ confined to the inner core was by far the least successful $Q_{\kappa}$ parameterization of that study. Fig. 6 shows the IC $Q_{\kappa}$ resulting from that experiment, as well as those of PREM and PAR3P, to be extremely unlikely and at least two orders of magnitude smaller than the range favored by the data. Given that the covariance between inner and outer core $Q_{\kappa}$ is less than $50 \%$ (Fig. 2), their $2-\sigma$ ranges are sufficiently far apart to make it almost certain that we are resolving two distinct regimes of bulk attenuation in the core. 
Combining our high inner core $Q_{\kappa}$ with our range of acceptable inner core $Q_{\mu}$ shows our results to be consistent with the range of 200-600 for upper inner core compressional attenuation $\left(Q_{\alpha}\right)$ from body waves [10]. In PREM, consistency with body waves was provided by the low $Q_{\kappa}$ value rejected by our forward modeling approach. For the upper inner core, $L=(4 /$ $3)\left(v_{\mathrm{S}} / v_{\mathrm{p}}\right)^{2}$ is approximately 0.135 and, if $Q_{\kappa}$ is large (as in QL6 and our models), $Q_{\alpha}$ is approximated by $Q_{\mu} / L$ [12]. For the QL6 lower limit of $Q_{\mu}=95$, this gives

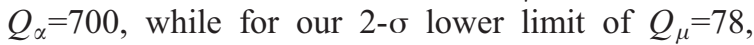
$Q_{\alpha}=578$. Thus, the inconsistency between body wave and free oscillation core attenuation constraints no longer appears as severe as previously feared.

Further, it must be recalled that this is an average over the entire inner core and, if $Q_{\alpha}$ increases with depth [31], more compressional attenuation is permitted at the top of the IC. When we test this by splitting the IC into two layers, we observe that an increase with depth is slightly favored and $Q_{\alpha}=450$ is allowed at the $1-\sigma$ model error level in the upper layer. Unfortunately, the tradeoffs between core parameters become large enough in this case that we are reluctant to draw further conclusions.

\section{Conclusions}

Through using the latest long-period $Q$ data and creating detailed model probability density functions based on fits to these data, we have been able to make a number of significant new observations: (a) the inconsistency between surface wave and free oscillation fundamental mode $Q$ measurements becomes less significant for model results when high-quality measurements of the Coriolis coupling band (300-600 s) are employed; (b) 2D PDFs confirm that a drop in $Q$ in the lower half of the lower mantle is even more likely than is the increase in $Q$ in the lower half of the transition zone; (c) $Q_{\kappa}$ resolution is good enough to show that modest but significant bulk attenuation is likely everywhere but in the inner core; and (d) improved model uncertainties show that inconsistencies between body wave and free oscillation constraints on inner core attenuation are not as great as previously believed. A more general result is that our approach to the global 1D inverse problem produces more useful models than those from traditional inversions. This encourages the extension of the approach to the joint 1D inverse problem for (frequency-dependent) attenuation, seismic velocities and density with data from body waves as well as free oscillations and surface waves.

\section{Acknowledgements}

This research has been supported by the Dutch National Science Foundation under grant number NWO:VICI 865.03.007. We wish to thank Gabi Laske and Guy Masters of Scripps IGPP, University of California, San Diego, for their work in maintaining the Reference Earth Model (REM) internet site and, in particular, the long-period seismic database stored there.

\section{References}

[1] S.-I. Karato, Importance of anelasticity in the interpretation of seismic tomography, Geophys. Res. Lett. 20 (1993) $1623-1626$.

[2] R. Woodward, A. Forte, W.-J. Su, A. Dziewonski, Constraints on the large-scale structure of the earth's mantle, in: Geophys. Monogr. Ser., vol. 74, AGU, Washington, D.C, 1993, pp. 89-109.

[3] A. Dziewonski, D. Anderson, Preliminary reference Earth model, Phys. Earth Planet. Inter. 25 (1981) 25297-25356.

[4] R. Widmer, G. Masters, F. Gilbert, Spherically symmetric attenuation within the earth from normal mode data, Geophys. J. Int. 102 (1991) 541-553.

[5] J. Durek, G. Ekström, A radial model of anelasticity consistent with long-period surface-wave attenuation, Bull. Seismol. Soc. Am. 86 (1996) 144-158.

[6] J.S. Resovsky, J. Trampert, Reliable mantle density error bars: an application of the neighbourhood algorithm to normal mode and surface wave data, Geophys. J. Int. 150 (2002) 665-672.

[7] G. Masters, G. Laske, On bias in surface wave and free oscillation attenuation measurements, EOS Trans.-Am. Geophys. Union 78 (1997) F485.

[8] J. Durek, G. Ekström, Investigating discrepancies among measurements of traveling and standing wave attenuation, J. Geophys. Res. 102 (1997) 24529-24544.

[9] G. Roult, E. Clevede, New refinements in attenuation measurements from free-oscillation and surface-wave observations, Phys. Earth Planet. Inter. 121 (2000) 1-37.

[10] B. Romanowicz, J. Durek, Seismological constraints on attenuation in the earth: a review, in: Geophys. Monogr. Ser., vol. 117, AGU, Washington, D.C, 2000, pp. 161-179.

[11] E.A. Okal, B.-G. Jo, Q measurements for phase x overtones, Pure Appl. Geophys. 132 (1990) 331-362. 
[12] D. Anderson, R. Hart, Q of the earth, J. Geophys. Res. 83 (1978) 5869-5882.

[13] J.H. Woodhouse, F.A. Dahlen, The effect of a general aspherical perturbation on the free oscillations of the earth, Geophys. J. R. Astron. Soc. 53 (1978) 335-354.

[14] G. Backus, J. Gilbert, Numerical applications of a formalism for geophysical inverse problems, Geophys. J. R. Astron. Soc. 13 (1967) 247-276.

[15] G. Backus, J. Gilbert, The resolving power of gross earth data, Geophys. J. R. Astron. Soc. 16 (1968) 169-205.

[16] J.S. Resovsky, M.H. Ritzwoller, Regularization uncertainty in density models estimated from normal mode data, Geophys. Res. Lett. 26 (1999) 2319-2322.

[17] M. Sambridge, Geophysical inversion with a neighbourhood algorithm: II. Appraising the ensemble, Geophys. J. Int. 138 (1999) $727-746$.

[18] A.N. Tikonov, V.Y. Arsenin, Solutions of ill-posed problems, John Wiley, New York, 1977.

[19] M. Sambridge, Geophysical inversion with a neighbourhood algorithm: I. Searching a parameter space, Geophys. J. Int. 138 (1999) 479-494.

[20] J.S. Resovsky, J. Trampert, Using probabilistic seismic tomography to test mantle velocity-density relationships, Earth Planet. Sci. Lett. 215 (2003) 121-134.

[21] G. Laske, G. Masters, http://mahi.ucsd.edu/gabi/rem.dir/ rem.home.html.
[22] J. Durek, M. Ritzwoller, J. Woodhouse, Constraining upper mantle anelasticity using surface wave amplitudes, Geophys. J. Int. 114 (1993) 249-272.

[23] J.J. Durek, Anelastic structure of the mantle from long period seismic data. PhD thesis, Harvard University (1994).

[24] B. Romanowicz, A global model of shear attenuation in the upper mantle, J. Geophys. Res. 100 (1995) 12375-12394.

[25] M. Smith, G. Masters, Aspherical structure constraints from free oscillation frequency and attenuation measurements, J. Geophys. Res. 94 (1989) 1953-1976.

[26] X.-D. Li, Asphericity of the earth from free oscillations, $\mathrm{PhD}$ thesis, Harvard University (1990).

[27] X. He, J. Tromp, Normal-mode constraints on the structure of the Earth, J. Geophys. Res. 101 (1996) 20053-20082.

[28] J.S. Resovsky, M.H. Ritzwoller, New and refined constraints on three-dimensional Earth structure from normal modes below 3 mHz, J. Geophys. Res. 103 (1998) 783-810.

[29] J.S. Resovsky, R. Pestana, Improved lower mantle Vp constraints from spectral fitting of normal mode data, Geophys. Res. Lett. 30 (2004) 1383-1386.

[30] J.-P. Montagner, B.L. Kennett, How to reconcile body-wave and normal-mode reference earth models, Geophys. J. Int. 125 (1996) 229-248.

[31] A. Souriau, P. Roudil, Attenuation in the uppermost inner core from broadband geoscope pkp data, Geophys. J. Int. 123 (1995) 572-587. 